\title{
A Design of Ultra-wideband Filter with Band-notched Characteristic Based on Left - Handed Materials
}

\author{
LI Shun-li ${ }^{1, a}$, ZENG Wen-bo $^{2}$ \\ ${ }^{1}$ School of Electric and Information Engineering, Guangxi University of Science and Technology ,545006, Liuzhou, \\ Guangxi,P. R. China \\ ${ }^{2}$ School of Electric and Information Engineering, Guangxi University of Science and Technology, 545006, Liuzhou, \\ Guangxi,P. R. China
}

\begin{abstract}
Based on the concept of left-handed material (LHM), a novel ultra-wideband (UWB) planar filter with negative permittivity and permeability was designed and proposed in this paper. By etching a cross-coupled structure on the surface of substrate, the filter achieved a frequency pass band of $2.86-11.22 \mathrm{GHz}$, which meets requirement of the UWB applications. Moreover, a notch frequency band of $7.26-9.13 \mathrm{GHz}$ also achieved simply by increasing the length of the left hand cross side structure. HFSS software was used to design and optimise the filter .The subtract of the filter is with a relative dielectric constant of 2.55 and the overall size of the filter is $7.6 \times 16 \times 0.8 \mathrm{~mm}^{3}$.
\end{abstract}

\section{Introduction}

Left - Handed Materials(LHM) refers to those class of materials which electric field E, magnetic field $\mathbf{H}$ and wave vector $\mathbf{K}$ satisfy left-hand rule in the electromagnetic wave propagation[1]. LHM has a series of bizarre physical phenomena, such as inverse Focus / divergence phenomenon, the phenomenon of negative refraction, inverse Cherenkov radiation effects, inverse Doppler effect, the inverse Gus - Han Sheen displacement and perfect lenses[2]. Because of these unusual electromagnetic properties, LHM has been named backward wave materials (BWM) and negative index of refraction materials (NIP) in the international community. Moreover, because of its negative permittivity $\varepsilon$ and permeability $\mu$, LHM was also been called meta-material or double negative material (DNG) [3]. LHM is currently only obtained artificially, but it attracts more and more researcher's attentions for its special properties at present[4].

In recent years, ultra-wideband (UWB) has attracted significant attention in wireless communication, networking, radar detection, and other applications [5].The UWB filter based on left handed materials is also a hot topic in recent years. The UWB frequency band is from 3.1 to $10.6 \mathrm{GHz}$. However, there still remains other narrowband wireless communication signals within the UWB spectrum, such as X-band (7.25 - 7.75 GHZ,7.9 - 8.4 GHZ), which may cause several frequency interference to the UWB communication system. In order to avoid and solve the problem caused by this frequency band, UWB filter with band-notched function for UWB applications are required[6].Many UWB filter with band-notched characteristic have been designed and proposed in the past few years, these filter usually with the structures such as adding a single minor sides in the

a Corresponding author: ZWB@gxut.edu.cn

(C) The Authors, published by EDP Sciences. This is an open access article distributed under the terms of the Creative Commons Attribution License 4.0 (http://creativecommons.org/licenses/by/4.0/). 
feeder, introducing slit structure in the filter, etc[7].In this paper , a UWB planar filter based on left-handed material (LHM) concept was designed, furthermore, by increasing the length of the cross structure on the LHM side of the subtract, a frequency notch-band from $7.26-9.13 \mathrm{GHz}$ is achieved.

\section{Principle of left - handed materials filter}

The characteristic of electromagnetic wave propagation in the medium are decided by dielectric constant $\varepsilon$ and permeability $\mu$ [8].In homogeneous isotropic material medium, single frequency wave phase constant $k$ and angular frequency $\omega$ satisfies the relation as

$$
k^{2}=\frac{\omega^{2}}{c^{2}} n^{2}
$$

where in, $n$ as the refractive index of the material and can be written as

$$
n^{2}=\varepsilon \mu
$$

Usually, permittivity and permeability are positive in the case of right hand material without loss. However , one can see from above expression, $n$ keeps the same when $\varepsilon$ and $\mu$ change sign simultaneously, this means that although the left-handed material does not exist in the nature, it theoretically consistent with the laws of physics.

In this paper, Smith parameter extraction method is used to verify the value of $\varepsilon$ and $\mu$. Suppose the filter substrate thickness is $h$, and the definition of transmission matrix $\mathrm{T}$ is expressed as

$$
T=\left(\begin{array}{cc}
\cos (n k d) & -z /[k \sin (n k d)] \\
k /[z \sin (n k d)] & -z /[k \sin (n k d)]
\end{array}\right)
$$

where in, $n$ is the refractive index, $z$ is the wave impedance, $k$ is the free space wave number.

In accordance with the transfer matrix and dispersion relations, $n$ can be written as

$$
\begin{aligned}
& n=\frac{1}{k h} \cos ^{-1}\left[\frac{1-S_{11}^{2}+S_{21}^{2}}{2 S_{11}}\right] \\
& \mu=n z
\end{aligned}
$$

where in, $z=\sqrt{\frac{\left(1+S_{11}\right)^{2}-S_{21}^{2}}{\left(1-S_{11}\right)^{2}-S_{21}^{2}}}$

Based on the analysis above, one can find from the expression of $\varepsilon$ and $\mu$ that the free-space wave number and the thickness of the substrate are higher than zero, so $k$ and $h$ can be ignored while determine the plus-minus of $\varepsilon$ and $\mu$. Furthermore, the $\varepsilon$ and $\mu$ vary from frequency can be obtained based on the simulation value of $S_{11}$ and $S_{21}$ as well as the application of MATLAB software, as shown in FIG 1.

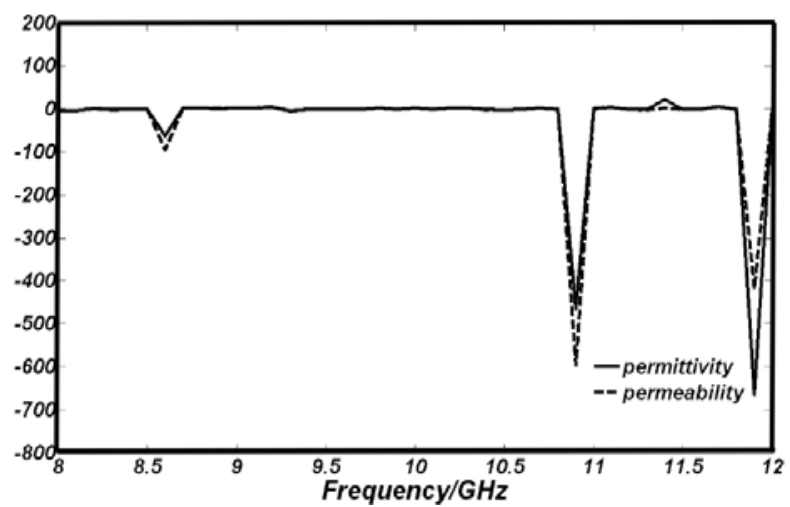

Figure 1. permittivity $\varepsilon$ and permeability $\mu$ vary from frequencies 
One can see from Figurel that the permittivity $\varepsilon$ and permeability $\mu$ can be negative simultaneously within a certain frequency range, thus means the designed filter achieve a negative refractivity therefore in accordance with the properties of left-handed materials.

\section{Design of the filter}

The UWB filter based on the concept of LHM proposed is etched on both sides of a piece of FR4 subtract, and the subtract is with a relative dielectric constant of $\varepsilon_{\mathrm{r}}=2.55$, dielectric loss tangent $\tan \delta$ $=0.0019$, and a thickness of $0.8 \mathrm{~mm}$. One side of the FR4 subtract is etched as a coplanar waveguide while the other side is etched as a high impedance micro-strip curved lines. Figure 2 shows the structure of the proposed filter .

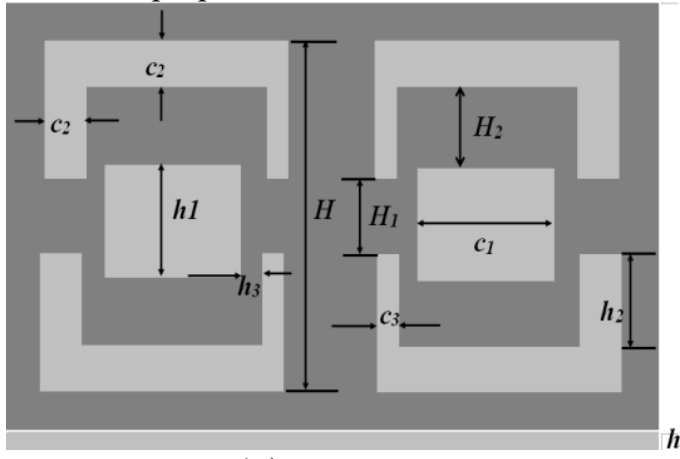

(a)

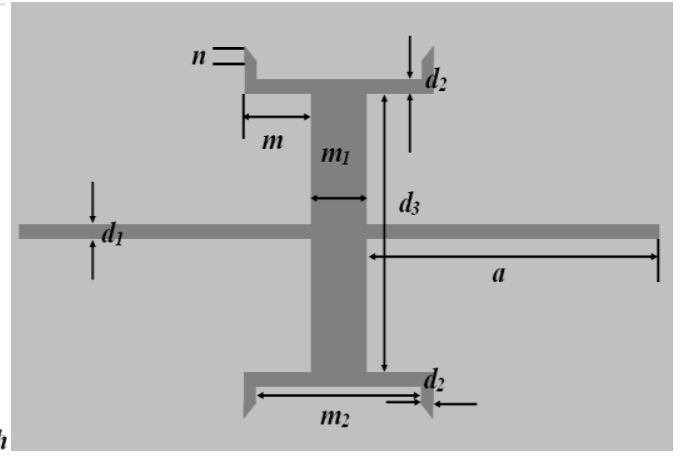

(b)

Figure 2. Structure of the proposed UWB filter: (a) top view and (b) bottom view.

The overall size of the filter is $16 \times 7.6 \times 0.8 \mathrm{~mm}^{3}$.HFSS software is used to design and optimise the filter. After intense work, geometrical parameters of the filter can be obtained as: $H=4.94 \mathrm{~mm}, H_{l}$ $=2 \mathrm{~mm}, c_{1}=2.2 \mathrm{~mm}, c_{2}=1 \mathrm{~mm}, c_{3}=1 \mathrm{~mm}, h_{1}=1.6 \mathrm{~mm}, h_{2}=0.47 \mathrm{~mm}, \mathrm{a}=7.39 \mathrm{~mm}, d_{1}=0.16 \mathrm{~mm}$, $d_{2}=0.22 \mathrm{~mm}, d_{3}=5.7 \mathrm{~mm}, m=1.75 \mathrm{~mm}, m_{l}=0.5 \mathrm{~mm}, m_{2}=3.56 \mathrm{~mm}$.

The return loss $\mathrm{S}_{11}$ and the insertion loss $\mathrm{S}_{21}$ can be obtained by HFSS software, as shown in Fig 3 . Frequency $/ \mathrm{GHz}$

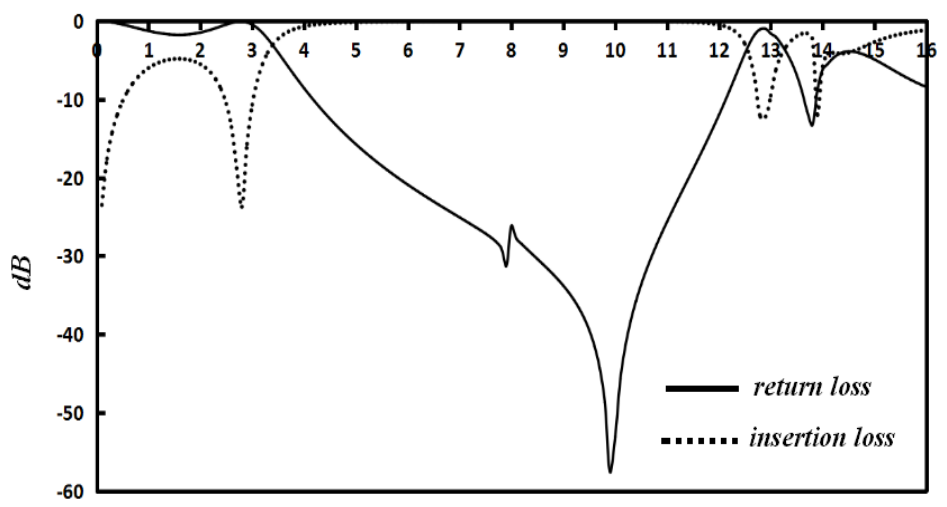

Figure 3. The return loss $S_{11}$ and the insertion loss $S_{21}$ of the proposed filter

To improve the performance of the filter and avoid electromagnetic interference from the $\mathrm{X}$ band (7.25 - 7.75GHZ, 7.9 - 8.4GHZ) within the UWB frequency band, a novel structure is introduced. By increasing the left hand cross side length of the material of the planar filter structure, the degree of coupling is increased and the frequency notched-band from 7.26-9.13 GHz can be achieved. The band-notched structure is shown as Fig 4 with geometrical parameters of $m=7.25 \mathrm{~mm}$ and $m_{3}=4 \mathrm{~mm}$ while other geometrical parameters fixed. 


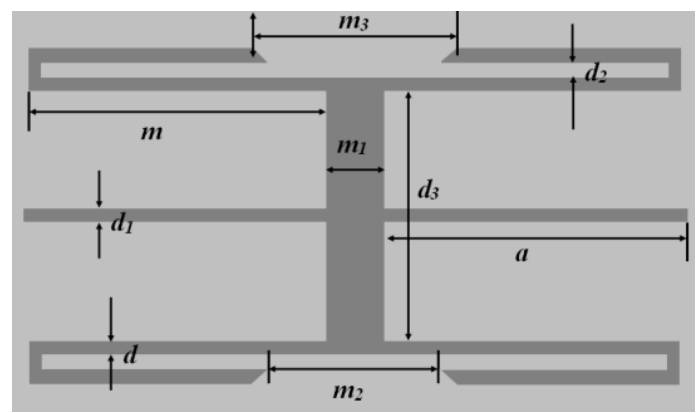

Figure 4. The bottom view of filter with band-notched characteristic

\section{Optimization and Analysis}

HFSS simulations show that the length of $m$ and $m_{l}$ have a great influence on the performance of the filter. In order to optimize the structure of the filter, the insertion loss $S_{21}$ is to be set as the destination function, and the $m$ and $m_{l}$ change while other parameters keep fixed. The simulation $\mathrm{S}_{21}$ of the proposed filter with different $m$ and $m_{l}$ are shown in Figure 5.

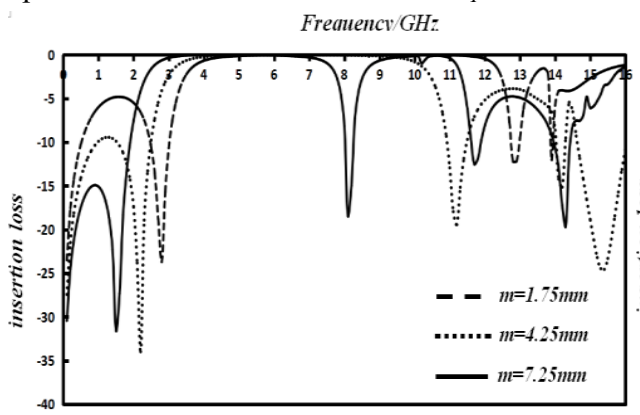

(a)

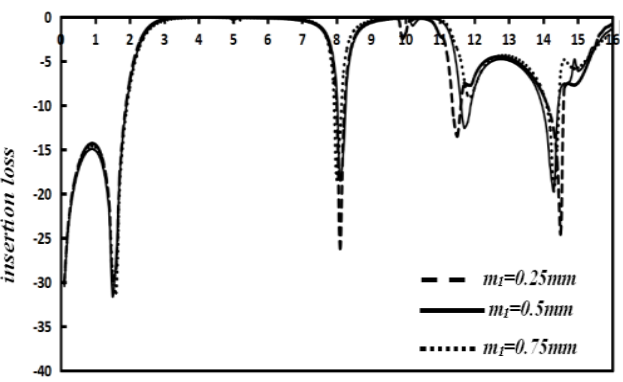

(b)

Figure 5. Simulation $\mathrm{S}_{21}$ of the proposed filter with different ( a ) $m$, ( b ) $m_{l}$

As one can be seen from the figure 5 , the notch frequency range varies when $m$ changes, according to the requirement of the size of filter, an ultimately $m=0.75 \mathrm{~mm}$ is adopted .Simulations indicate that $m_{l}$ mainly affects the range pass-band of the filter, and the pass-band of the filter gradually widened with an increasing $m_{l}$, but when the value of $m_{l}$ is greater than $0.5 \mathrm{~mm}$, the pass-band trends to narrow, then an optimized $m_{l}=0.5 \mathrm{~mm}$ is adopted. Simulations also indicate that $m_{l}$ mainly affects the coupling efficiency of the filter and therefore affects the insertion loss, while $m$ mainly affect impedance of the micro-strip line and therefore affects return loss of the filter[9].

Frequency $/ \mathrm{GHz}_{z}$

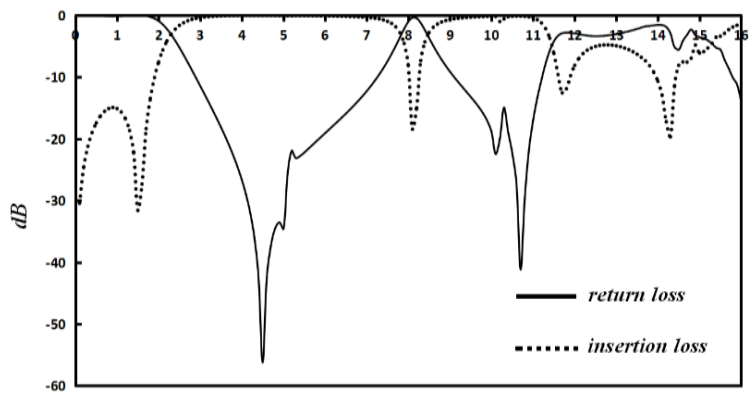

Figure 6.Simulation return loss and insertion loss of the UWB filter with band-notched characteristic based on LHM 
The return loss $S_{11}$ and insertion loss $S_{21}$ of the proposed filter is shown in Figure 6, a pass-band from 2.86-11.22 GHz with $\mathrm{S}_{11}$ larger than $-10 \mathrm{~dB}$ and a notch frequency band of 7.26 - 9.13 $\mathrm{GHz}$ with $\mathrm{S}_{21}$ less than $-10 \mathrm{~dB}$, are achieved.

\section{Conclusion}

Based on the concept of left-handed materials ,this paper present a novel design of UWB filter with a notch frequency band from $7.26-9.13 \mathrm{GHz}$, which results the filter a good performance of rejecting the electromagnetic interference caused by the X-band signals. The return loss of the filter on the resonance point achieves $-56.21 \mathrm{~dB}$ and the gain is close to 1 . Moreover, the filter is compact with an overall size of $7.6 \times 16 \times 0.8 \mathrm{~mm}^{3}$, it has broad development and application prospects in the field of UWB applications.

\section{Acknowledgement}

The authors would like to acknowledge the financial support from the projects of Natural Science Fou ndation of Guangxi (NO.2015GXNSFAA139289), and Key Laboratory of Cognitive Radio and Infor mation Processing, Ministry of Education (Guilin University of Electronic Technology) ( No. CRKL1 50205)

\section{References}

1. J.-Q. Huang,Q.-X. Chu.COMPACT UWB BAND-PASS FILTER UTILIZING MOD-IFIED COMPOSITE RIGHT/LEFT-HANDED STRUC-TURE WITH CROSS COUPLING[J].PIER, 8(2010).

2. Shah Nawaz Burokur, Mohamed Latrach.Left-Handed Medium effect on the characteristics of a circular patch antenna.IEEE,11(2007).

3. W.-B. Zeng, J. Zhao, and Q.-Q. Wu, Compact planar ultra-wideband wide-slot antenna with an assembled band-notched structure,MICROW OPT TECHN LET,7(2012).

4. ZHU Xiao-ming.Dsihn of miniaturization band-notched UWB antenna,Journal of Heilongjiang Institute of Technology,1(2014).

5. Wu Yiwei,Li Simin,An microstrip patch antenna based on left-handed material,Journal of Guilin University of Electronic Technology,6(2013).

6. K. Bahadori, Y. Samii, "A miniaturized elliptic-card UWB antenna with WLAN band rejection for wireless communications", IEEE,11(2007).

7. C. Yoon, W.-J. Lee, W.-S. Kim, H.-C. Lee, and H.-D. Park, Compact band-notched ultra-wideband printed antenna using inverted L-slit, MICROW OPT TECHN LET,1(2012).

8. X.-L. Liu, Y.-Z. Yin, P.-A. Liu, J.-H. Wang, and B. Xu, A CPW-fed dual band-notched UWB antenna with a pair of bended dual-L-shape parasitic branches,PIER,6(2013).

9. M. T. Islam, R. Azim, and A. T. Mobashsher, Triple band-notched planar UWB antenna using parasitic strips, PIER,4(2012). 